\title{
VOID COEFFICIENT SENSITIVITY ANALYSIS FOR THE TRIGA MARK II REACTOR AT L.E.N.A. (UNIPV)
}

\author{
D. Portinari ${ }^{1,4}$, A. Cammi ${ }^{1}$. S. Lorenzi ${ }^{1}$, M. Aufiero ${ }^{3}$, Y. Calzavara ${ }^{4}$, A. Bidaud ${ }^{2}$ \\ ${ }^{1}$ Politecnico di Milano; Dept. of Energy, Nuclear Eng. Division, Via La Masa 34, 20156, Milan, Italy \\ ${ }^{2}$ LPSC-IN2P3-CNRS; 53 Rue des Martyrs, 38026 Grenoble, France \\ ${ }^{3}$ Milano Multiphysics; Polihub, Politecnico di Milano, via Durando 39, 20158 Milano, Italy \\ ${ }^{4}$ Institut Laue Langevin; 71 Rue des Martyrs, 38042 Grenoble, France
}

\begin{abstract}
Sensitivity analysis studies the effect of a change in a given parameter to a response function of the system under investigation. In reactor physics, this usually translates into the study of how cross sections and fission spectrum modifications affect the value of the multiplication factor, the delayed neutron fraction or the void coefficient for example. Generalized Perturbation Theory provides a useful tool for the assessment of adjoint weighed functions such as $k_{e f f}$ and void coefficient sensitivities. In this work, the capability of SERPENT code to perform sensitivity calculation based on GPT is used to study the TRIGA Mark II research reactor installed at L.E.N.A. of University of Pavia. A general sensitivity analysis to the most important reactor's cross sections has been performed in order to highlight the biggest reactivity contributions. Two numerically challenging tasks related to GPT calculation have been performed thanks to the relatively quick Monte Carlo approach allowed by this reactor: investigating the linearity of the reactivity injection caused by the flooding of the central channel, and calculating the fuel void coefficient sensitivity to the coolant density.
\end{abstract}

KEYWORDS: SENSITIVITY, PERTURBATION THEORY, MONTECARLO, TRIGA

\section{INTRODUCTION}

The Triga Mark II is a research reactor designed by General Atomics, widely employed among Universities and Research Centers for its versatile applications. A matrix of $U-\mathrm{ZrH}_{2}$ fills the fuel rods $(3.58 \mathrm{~cm}$ in meat diameter and $35.35 \mathrm{~cm}$ in length) that are disposed in concentric rings to constitute the core of the reactor pool (Figure 1). The Hydrogen inside the fuel provides most of the moderation along with the light water in the pool. The coolant flows by natural circulation through the active length to absorb a nominal power that, in the L.E.N.A. case, is $250 \mathrm{~kW}$. The reflector is a graphite wall that surrounds the pool and provides a thermal column for the extraction of neutrons. A slightly epithermal spectrum is one of the consequences of the moderation taking place inside the fuel: the Hydrogen atoms have a quantized energy fixed to multiples of 0.13 $\mathrm{eV}$, while the Thermal energy is $0.025 \mathrm{eV}$. This phenomenon is quite unique and gives the fuel a strongly negative temperature feedback coefficient that allows the reactor to endure very sharp reactivity transients (pulse operation), without any extra safety measure. The short-term control of the reactor is provided by a Regulating Control Rod inserted in one of the outer rings of the core, 
while a Transient Control Rod pneumatic insertion provides the shutdown margin at anytime. A third Shim Control Rod is operated in one of the inner rings for long-term reactivity compensation. All the control rods are made of boron carbide B4C, $2.52 \mathrm{~g} / \mathrm{cc}$ density.

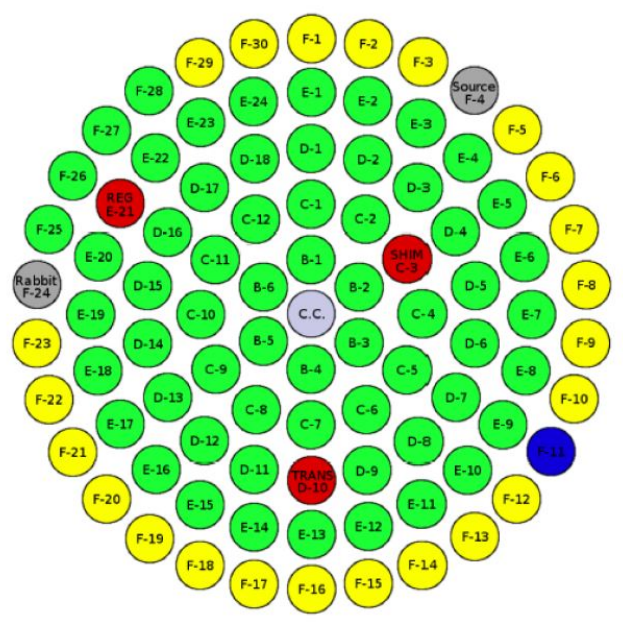

Figure 1: Triga Mark II Reactor element disposition: fuel rods (green), control rods (red), graphite reflector rods (yellow), experimental/source channels (grey), empty slots (blue)

The in-fuel moderation makes the thermalization process very effective and the strongly negative value of the fuel feedback coefficient plays a key role in the reactor's neutronics. This coefficient can be estimated under isothermal approximation by integrating the power curve in a specific transient and assuming that all the energy gets stored by the fuel thermal capacity [1]. By doing so, the contribution of the coolant to the fuel feedback coefficient is neglected. Adopting perturbation methods, it is possible to assess the impact of the coolant density to the fuel void coefficient, which accounts for density and composition perturbations in the fuel, while temperature associated perturbations must be handled separately. Generalized Perturbation Theory (GPT) applied to neutron transport requires the calculation of neutron's importance through the adjoint flux, which corresponds to the contribution of each neutron to reactivity or to some reaction rates [2].

It has been recently shown that also Monte Carlo codes can calculate $k_{\text {eff }}$ sensitivity coefficients by weight processing in the collision history, exploiting the capabilities of Iterated fission probability (IFP, [3]) . Sensitivities of kinetics parameters can be calculated as well. [4]. In this work, the ability of the SERPENT2 code to perform sensitivity calculation based on GPT is used to study the TRIGA Mark II research reactor installed at L.E.N.A. of University of Pavia. A general sensitivity analysis of the most important reactor's cross sections has been performed in order to highlight the biggest reactivity contributions. The main objective of the study has been exploiting the SERPENT code to tackle two numerically challenging tasks with GPT. The first one is investigating the linearity of the reactivity injection caused by the flooding of the central channel. The second one is the calculation of the void coefficient sensitivity of the fuel, a second derivative of $k_{e f f}$ that can become very helpful in assessing the uncertainty on the fuel composition. Finally, an analysis on the convergence speed of the GPT tool is reported in order to highlight the accessibility of the different sensitivity coefficients 


\begin{tabular}{|c|c|c|}
\hline Perturbed cross section & Perturbed nuclide & $k_{\text {eff }}$ sensitivity \\
\hline \hline Fission & U-235 & +0.356 \\
\hline Capture & U-235 & -0.119 \\
\hline Total & U-235 & $\mathbf{+ 0 . 2 3 7}$ \\
\hline Scattering & U-238 & +0.006 \\
\hline Fission & U-238 & +0.029 \\
\hline Capture & U-238 & -0.038 \\
\hline Total & U-238 & $\mathbf{+ 0 . 0 0 3}$ \\
\hline Scattering & H-1 & +0.133 \\
\hline$S(\alpha, \beta)$ & H-1 & +0.028 \\
\hline Capture & H-1 & -0.056 \\
\hline Total & H-1 & $\mathbf{+ 0 . 1 0 5}$ \\
\hline
\end{tabular}

Table 1: $k_{e f f}$ sensitivity coefficients to perturbations in fuel material cross sections

\section{SENSITIVITY ANALYSIS}

The main $k_{e f f}$ sensitivities for the Triga Reactor are shown in Table 1 and 2. It is evident that the most sensitive nuclides are Uranium 235 and Hydrogen inside the fuel. The relative uncertainty on the estimation of these coefficients is always $<1 \%$ after 500 cycles of 200000 neutron hisotries (16 virtual nodes for a total of 130GB of RAM, jeff311 libraries employed). The coolant sensitivity is much lower with respect to the fuel one. This is due to the well known compensation of scattering and capture contributions in the moderation, that by canceling each other bring down the total coefficient value. Employing the water sensitivities it is possible to successfully compare the density component of the feedback coefficient for the coolant with the one obtained by direct MCNP benchmark simulation $\left(\alpha_{\text {coolant }}=-2.4 \mathrm{pcm} / \mathrm{K}\right.$ at $300 \mathrm{~K}$ [5]), employing the coolant temperature expansion coefficient $\beta$ :

$$
\alpha_{\text {coolant }}=S_{H_{2} O}^{k} \cdot \beta_{\text {exp }}=-2.1 p c m / K
$$

\section{CENTRAL CHANNEL FLOODING}

The central channel of the TRIGA reactor is void, but a set of water filled cylinders $(1.5 \mathrm{~cm}$ diamater. 17 and $18 \mathrm{~cm}$ length) can be inserted (Figure 2) in order to study the reactivity worth of its flooding, i.e. the opposite of its void coefficient. The reactivity was obtained by comparison of the flooded and unflooded critical control rod position, where the the differential reactivity of the control rod is known. The experimental position of the control rod yields a reactivity spike of 43 


\begin{tabular}{|c|c|c|}
\hline Perturbed cross section & Perturbed nuclide & $k_{\text {eff }}$ sensitivity \\
\hline \hline Scattering & H-1 & +0.186 \\
\hline Capture & H-1 & -0.125 \\
\hline Total & H-1 & $\mathbf{0 . 0 6 2}$ \\
\hline Scattering & O-16 & +0.036 \\
\hline Capture & O-16 & -0.001 \\
\hline Total & $\mathbf{O}-16$ & $\mathbf{+ 0 . 0 3 5}$ \\
\hline
\end{tabular}

Table 2: $k_{e f f}$ sensitivity coefficients to perturbations in coolant cross sections

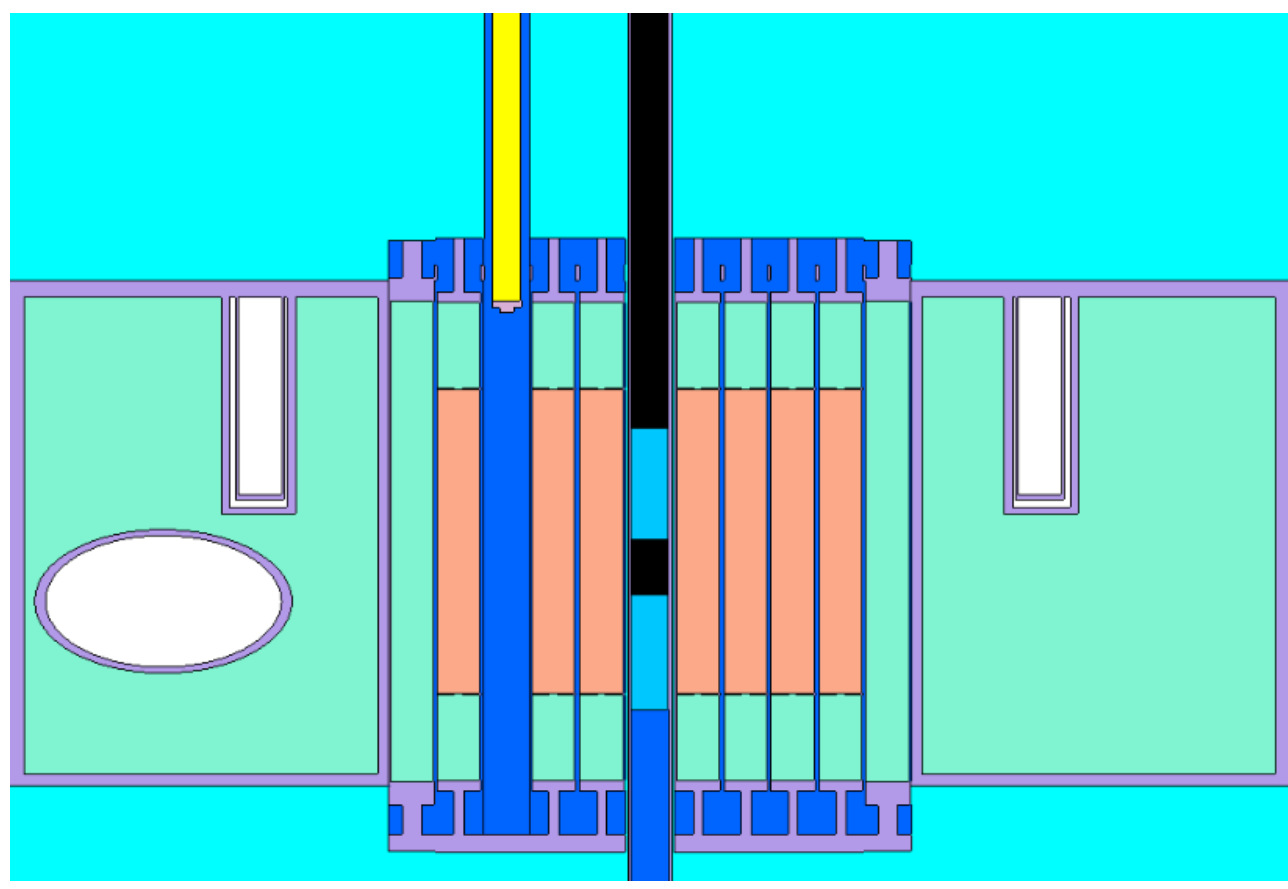

Figure 2: Vertical section of The TRIGA Mark II reactor model with water cylinders (light blue) inserted in the central channel (black), fuel rods (orange), control rod (yellow) and graphite reflector (green) 

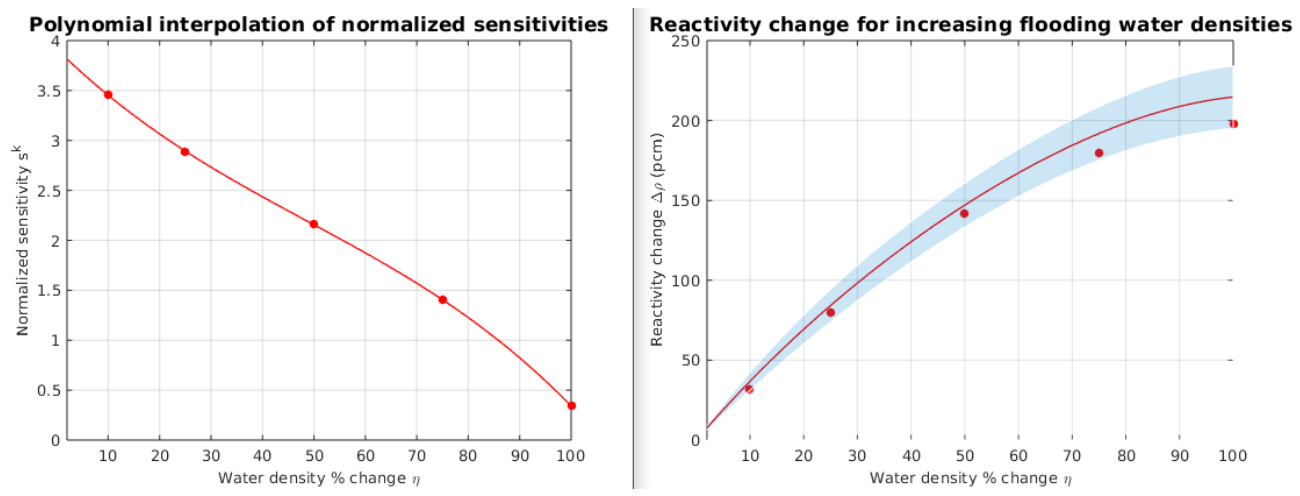

Figure 3: Central channel flooding analysis

pcm, while the direct SERPENT calculations yield a much bigger value. Even by exaggerating the role of the structural materials in the cylinders configuration, the result of both direct simulations and GPT estimations are not matching the experiments. Most often, the flooding reactivity doesn't have a linear behavior, because the water that is injected into the void channel proves to have a higher importance than the one that comes at the end of the supposed flooding process. In order to assess this non-linearity, the reactivity worth has been obtained for flooding water densities $N_{C C}$ of $10 \%, 25 \%, 50 \%$ and $100 \%$ the nominal one. The total sensitivity $S_{C C}^{k}$ of water inside the cylinders has been calculated in each point and then normalized to the parameter $\eta$ (percentage change of the nominal density) with eq. 2 . Since the biasing process is not yet implemented in SERPENT2, the statistics of this process is challenging because the volume where the tallies are scored is restricted to the central channel.

$$
s^{k}(\eta)=\frac{S_{C C}^{k}\left(N_{C C}\right)}{\frac{N_{C C}}{N_{C C}^{n o m}} \cdot 100}=\frac{S_{C C}^{k}(\eta)}{\eta}
$$

Interpolating the obtained points, a rough estimation of the trend of $s^{k}\left(N_{C C}\right)$ is obtained. This trend is an approximate derivative of the reactivity worth with respect to a percentage change in nominal density. Employing eq. 3 it is then possible to make a comparison between GPT results and direct calculations (Fig. 3).

$$
\Delta \rho(\eta)=\int s^{k}(\eta) d \eta
$$

Since the flooding of the central channel shows a non-linear behavior, we can confirm that the $\alpha_{v}$ obtained via experiment is indeed an average value over the central channel filling procedure. No changes of sign are highlighted, but a slight decrease in power has been recorded during the experimental flooding of the central channel. If this behavior was to be investigated with GPT, some negative sensitivities might be detected along the axial direction. 


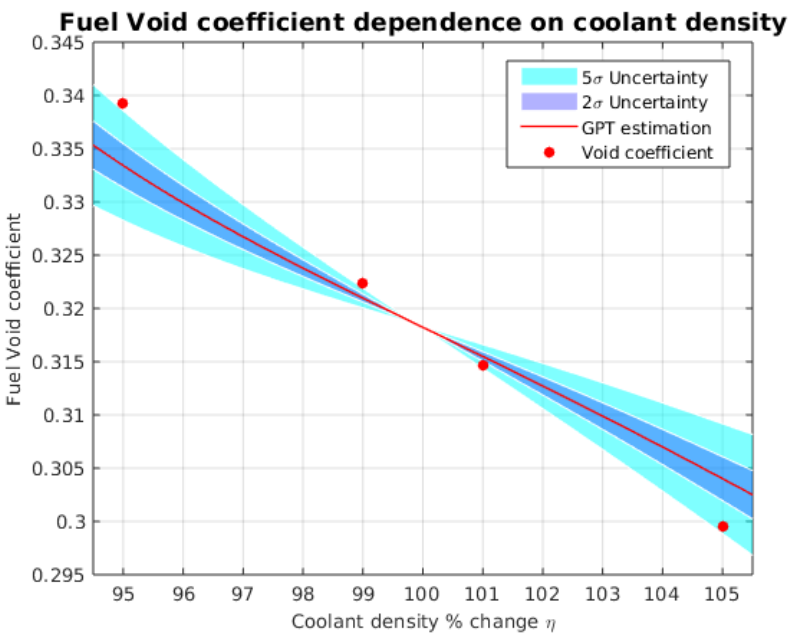

Figure 4: Fuel void coefficient analysis

\section{VOID COEFFICIENT SENSITIVITIES}

The void coefficient sensitivity calculation is very expensive from the computational point of view. In order to contribute to the tally, a neutron has to score in the material where the $\alpha$ coefficient is calculated, in the perturbed material, and cause a fission in the fuel. The TRIGA Reactor provides one of the few input files where a comparison between sensitivities of first and second order can be performed with reasonable computational cost (unlike other reactors with more complicated geometries [6]). The definition of "void coefficient" states that perturbations must be carried out on the total fuel cross section.

$$
\alpha_{f u e l}=S_{\Sigma_{\text {fuel }}}^{k_{\text {fuff }}}=\frac{\frac{d k}{k}}{\frac{d \Sigma_{\text {fuel }}}{\Sigma_{\text {fuel }}}}=\frac{<\Phi^{\dagger} \mid \Sigma_{\text {fuel }} \Phi>}{<\Phi^{\dagger} \mid \frac{1}{k} L_{T_{f}} \Phi>} \quad S_{\Sigma_{\text {cool }}}^{\alpha_{\text {fuel }}}=\frac{\frac{d \alpha_{\text {fuel }}}{\alpha_{\text {fuel }}}}{\frac{d \Sigma_{\text {cool }}}{\Sigma_{\text {cool }}}}
$$

While the atomic density has a direct impact on the total cross section, the Doppler effect and the quantized energies of hydrogen bring to discrete alterations in the moderation process at very specific lethargies of the neutrons. This means that the feedback coefficient can't be easily studied with GPT. Extended Generalized Perturbation Theory (xGPT) procedures might be envisaged in order to study the temperature feedback, focusing on $S(\alpha, \beta)$ perturbations in particular. For densities of light water of $95 \%, 99 \%, 101 \%$ and $105 \%$ the nominal one, both the $\alpha_{\text {fuel }}$ and its sensitivity $S_{\Sigma_{\text {cool }}}^{\alpha_{\text {fuel }}}$ to the coolant channel have been calculated. By interpolation of these sensitivities and further integration of the obtained curve, we can compare the results in Figure 4 much like it was done in section 3. A quite clear negative linear dependence is found, meaning that an expansion of the coolant brings to an increase of the fuel void coefficient. The result matches first order sensitivities only at $5 \sigma$, furthermore, a value of -0.3 for this sensitivity means that even a $50^{\circ} \mathrm{C}$ change in the coolant leads to change in void coefficient $<1 \%$. This dependency is related to the expansion effect, and while it is the main one for water, we can't say the same for the fuel. 

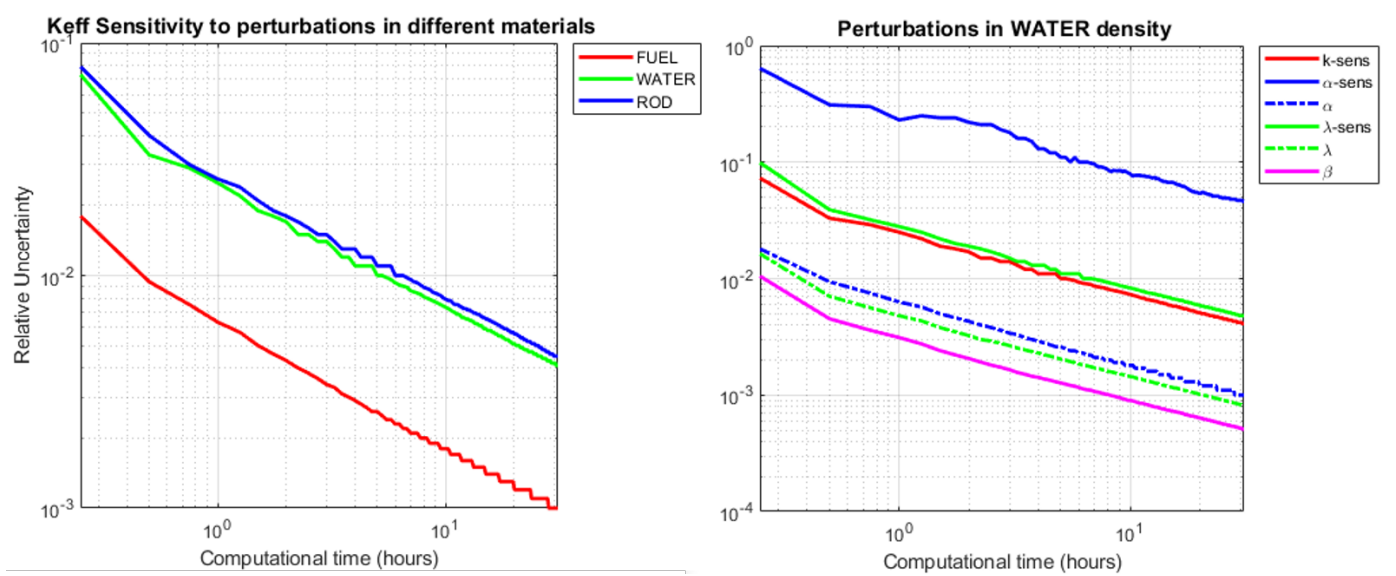

Figure 5: Convergence speed of the main sensitivity coefficients

\section{CONVERGENCE SPEED}

As already mentioned, sensitivity coefficients can be quite easy to obtain for the multiplication factor, while for the other parameters, especially the void coefficient, they can be slow to converge. This stems from the statistical evidence that the $k_{e f f}$ calculation involves way more neutrons with respect to the $\alpha_{v}$, whose estimation is based on the scoring of specific reactions for each neutron path. The TRIGA Reactor has a small core, whose moderation is very much optimized by the presence of hydrogen in the core. Since the sampling of scattering reactions is one of the main bottlenecks in Mont Carlo codes speed, leading to faster Monte Carlo simulations with respect to other reactor models. In Figure 5, the convergence speed of some sensitivity coefficients is compared to the one of other kinetics parameters. The void coefficient clear dependence on the coolant density is confirmed again by the converging trend in accordance with the other logarithmic derivatives. A peculiar feature of this reactor can be found in the relatively low sensitivity of the safety parameters to the perturbations in the control rod. In particular, the convergence of the $\lambda_{e f f}$ sensitivity is never reached because of the almost perfect compensation between the negative contributions given by B-10 capture and the positive one given by B-11 scattering. As a comparison, the same parameters are shown for the RHF research reactor [6], where the Nickel control rod doesn't have any scattering contribution, and on the other side shows a slower convergence for the void coefficient.

\section{CONCLUSIONS}

Sensitivity Analysis highlighted the main feature of the TRIGA Mark II reactor: low coolant feedback coefficient and high sensitivity coefficient for the fuel. The calculations on central channel flooding and fuel void coefficient were very helpful in testing the performance of the GPT algorithm in challenging conditions characterized by low statistical relevance. GPT was consistent with direct calculations in the flooding case and sensitivities of first and second order were successfully compared for the fuel material.

With a better understanding of the water cylinders injection experimental framework, the void co- 

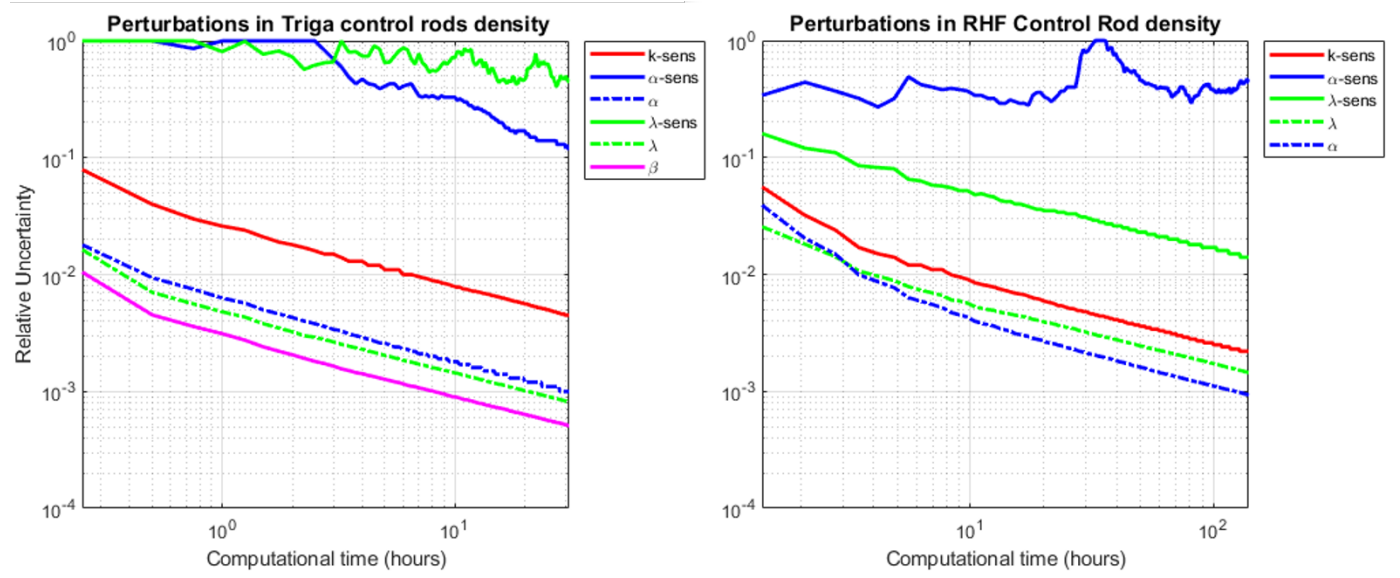

Figure 6: Comparison of convergence behavior for the TRIGA and the RHF control rods

efficient experiment could be simulated more accurately and the comparison could be extended to the radial dependence of the void coefficient. Even though no information was gathered on the fuel feedback coefficient, the dependence of fuel perturbations on coolant density was highlighted, and this might suggest an analogous behavior of the temperature coefficient, since they both directly affect the moderation process. The analysis on convergence speed shows that also the TRIGA coolant void coefficient was quite accessible from the computational point of view, and a dependency on the control rod density can suggest that the experimental setting of the boron absorbers might affect the results of the coolant channel flooding.

\section{REFERENCES}

[1] R. M. G. do Prado Souza and A. Z. Mesquita. "Measurements of the isothermal, power and temperature reactivity coefficients of the IPR-R1 TRIGA reactor." Progress in Nuclear Energy, volume 53(8), pp. 1126 - 1131 (2011). International nuclear Atlantic conference - INAC 2009.

[2] A. Gandini. "A generalized perturbation method for bi-linear functionals of the real and adjoint neutron fluxes." Journal of Nuclear Energy, volume 21(10), pp. 755-765 (1967).

[3] J. Leppanen, M. Aufiero, E. Fridman, R. Rachamin, and S. van der Marck. "Calculation of effective point kinetics parameters in the Serpent 2 Monte Carlo code." Annals of Nuclear Energy, volume 65, pp. 272-279 (2014).

[4] M. Aufiero, A. Bidaud, M. Hursin, J. Leppanen, G. Palmiotti, S. Pelloni, and P. Rubiolo. "A collision history-based approach to sensitivity/perturbation calculations in the continuous energy Monte Carlo code SERPENT." Annals of Nuclear Energy, volume 85, pp. 245-258 (2015).

[5] A. Cammi, M. Zanetti, D. Chiesa, M. Clemenza, S. Pozzi, E. Previtali, M. Sisti, G. Magrotti, M. Prata, and A. Salvini. "Characterization of the TRIGA Mark II reactor full-power steady state." Nuclear Engineering and Design, volume 300, pp. 308 - 321 (2016).

[6] D. Portinari, M. Aufiero, Y. Calzavara, A. Cammi, S. Lorenzi, and A. Bidaud. "Uncertainty and sensitivity analysis of safety parameters for High Flux Reactor at Institut Laue-Langevin." The International Conference on Mathematics and Computational Methods applied to Nuclear Science and Engineering (M\&C 2019) (2019). 